\title{
Dementia and antihypertensive treatment
}

\section{Willem H. Birkenhäger ${ }^{a}$, Françoise Forette ${ }^{b}$ and Jan A. Staessen ${ }^{c}$}

\begin{abstract}
Purpose of review
We present an updated overview on the long-term effects of hypertension on the occurrence of cognitive dysfunction and overt degenerative or vascular dementia later in life. The preventative effects of antihypertensive treatment in this regard are examined, with a focus on placebo-controlled, double-blind, randomized prospective trials.
\end{abstract}

\section{Recent findings}

The stereotypical straightforward linear relationship between mid-life hypertension and dementia later in life can no longer be considered strictly invariable. Successfully treated hypertensive patients who are still at risk for clinical dementia late in life may ultimately fare better in the presence of a slightly elevated rather than low systolic blood pressure. The mechanisms underlying this 'J-curve' phenomenon are currently being explored. Recently completed prospective randomized antihypertensive trials (Syst-Eur 2, PROGRESS and SCOPE) have yielded variable results, and merit cautious interpretation.

\section{Summary}

The incidence and prevalence of dementia are increasing exponentially worldwide, particularly in those older than 70 years. Because hypertension predisposes to dementia, therapeutic blood pressure titration should be maintained over the years, and intensified beyond 70 years in order to avoid over-treatment in the latter period.

\section{Keywords}

hypertension, cognitive decline, dementia prevention

Curr Opin Nephrol Hypertens 13:225-230. (c) 2004 Lippincott Williams \& Wilkins.

aErasmus University Rotterdam, Rotterdam, The Netherlands, 'Hopital Broca, Universite Rene Descartes, Paris, France and ${ }^{\mathrm{C} C a m p u s ~ G a s t h u i s b e r g, ~ L e u v e n, ~}$ Belgium

Correspondence to W.H. Birkenhäger, MD, PhD, FAHA, Emeritus Professor of Medicine, Erasmus University Rotterdam; private address: Karl Marxstr. 35, 3076 DP Rotterdam, The Netherlands.

Tel: +31 10 4325649; fax: +31 10 4325790; e-mail: w.birkenhager@chello.nl

Current Opinion in Nephrology and Hypertension 2004, 13:225-230

C 2004 Lippincott Williams \& Wilkins

$1062-4821$

\section{Introduction}

Roughly speaking, dementia is characterized by the development of a range of intellectual and related mental defects, such as progressive loss of memory, increasing disorientation in space and time, loss of autonomy, and emotional depersonalization. Hypertension has proven to be a major predisposing factor for the development of both vascular and degenerative (Alzheimer's) dementias, either following stroke or through more insidious microcerebrovascular processes, or both. Such sequelae of hypertension and the protective potential of antihypertensive (drug) treatment were covered in a series of recent reviews [1-6]. However, the accumulation of more recent epidemiological and therapeutic findings linking blood pressure, cognitive impairment and dementia creates the need for an updated review.

\section{Relative (ir)relevance of subtyping dementias in relation to hypertension as a risk factor}

Despite the extensive scientific knowledge of the differential clinical and neuroanatomical hallmarks of the two main dementia prototypes, namely Alzheimer's disease and vascular dementia, the importance of their discriminating degenerative and vascular characteristics has diminished in the face of integrated preventive medicine. According to Hofman et al. [7], the two subtypes share atherosclerosis as a major risk factor, which is reflected by the use of hypertension as a common denominator in several prospective, therapeutic long-term trials (discussed below).

From the neuropathological perspective, an elaborate and authoritative exploration, conducted under the auspices of the Medical Research Council in the UK [8], confirmed the existence of a common trait between the substrates of the subtypes of dementia. Given this considerable overlap between the two, it now seems both appropriate and feasible to consider dementia as one disease entity, at least for practical purposes at the population level. Such a pragmatic approach (with all due respect to the detailed literature inherited from the past century that separates the two subtypes) would greatly facilitate the design of future prospective trials on the prevention of dementias.

\section{Cognitive performance in relation to blood pressure}

According to a classic review of a dozen cross-sectional studies of the correlation between blood pressure and cognition [1], the results were far from uniform. 
Although differences in patient selection and methodology may well have been responsible for the variance in the observed relationships, it must be borne in mind that the time scale of the natural history of hypertension per $s e$ and the occurrence of its eventual sequela of cognitive deterioration tend to differ widely. Hypertension is mainly regarded as a mid-life phenomenon, whereas dementia tends to occur in those older than 70 years. Small wonder, then, that one must resort to longitudinal observations in order to correlate the conditions.

In this regard, Elias et al. [9] broke new ground in the Framingham cohort by examining cognitive function and memory performance in hypertensive persons 12-14 years after recruitment, in relation to their initial blood pressures. The majority of individuals (88\%) had remained untreated for hypertension in the interim. After correction for demographical variables and cardiovascular risk factors other than high blood pressure, four out of the eight implemented parameters of cognitive performance were found to be negatively correlated with initial systolic and diastolic blood pressures. In the Honolulu-Asia Aging Study [10], a similarly negative relationship was found between systolic blood pressure at enrolment and risk for cognitive impairment after an interval of 25 years.

In a meticulously conducted 15-year follow-up study, Skoog et al. [11] examined blood pressure and dementia in Gothenburg, Sweden. They analyzed the relation between blood pressure and dementia starting with nondemented hypertensive patients at age 70 years, and then observing them again at age 70-75 years, 75-79 years and up to 85 years. Patients who developed dementia in the final period of observation (i.e. age 7985 years) had higher systolic and diastolic blood pressures at age 70 years than did those who did not develop dementia. Remarkably, in the high-risk group, blood pressure in the years just before the onset of dementia tended to become lower than pressures in the latter group. (This feature is discussed below.) In another Swedish investigation, conducted by Kilander et al. [12], 999 men were first examined at age 50 years and followed up for 20 years. At age 70 years, cognitive function was highest in the group with an initial diastolic blood pressure below $70 \mathrm{mmHg}$ and lowest in those with a pressure greater than $105 \mathrm{mmHg}$. In the recently reported Sydney Older Persons Study [13], 377 nondemented persons aged 75 years and over were followed up for 6 years. Of the 263 survivors after that period, 63 $(16.7 \%)$ had developed dementia.

Hence, the trend identified from longitudinal studies suggests that hypertension from mid-life onward tends to predispose to the development of cognitive decline and dementia during the course of the eighth decade of life.
It should be added that this course is modified in hypertensive individuals who receive antihypertensive treatment [14-16].

\section{Evidence for a 'J-curve' relation between blood pressure and dementia}

In the previous section we referred to findings reported by Skoog et al. [11], in their carefully conducted followup study. Those investigators found that patients who were prone to become demented, on the basis of past hypertension, paradoxically exhibited lower blood pressures in the years just preceding the development of (mainly Alzheimer's) dementia than did those who kept well. White matter lesions on computed tomography also seemed to predispose to such a decline in blood pressure. Skoog [17] offered the hypothesis that failure to maintain usual blood pressure at this stage might well be an expression of Alzheimer-type lesions in prefrontal autonomic centres, resulting in central dysregulation of blood pressure. It may further be speculated that this could give rise to subsequent orthostatic falls both in blood pressure and in cerebral blood flow [18], which would probably accelerate the process of cerebrocellular damage.

In this regard, it may be worth mentioning the finding by Bellelli et al. [19] that increased diurnal blood pressure variability in elderly individuals (as an expression of autonomic dysfunction) appeared to be related to a low Mini-Mental State Examination score. Because that was a small series, with antihypertensive drug treatment as a potentially confounding factor, such data must be confirmed on a larger scale in untreated older hypertensive persons.

Irrespective of its putative mechanisms, this phenomenon may explain why occasional observations very late in life [20] revealed that low systolic pressures were an omen that preceded loss of cognition. In the 6-year Kungsholmen Project [16], it was a very low diastolic pressure $(<65$ versus $66-90 \mathrm{mmHg})$ that was associated with an increased relative risk for dementia, particularly in patients on antihypertensive treatment. By contrast, high systolic blood pressures $(>180 \mathrm{mmHg})$ carried a similar relative risk, whereas quite low systolic pressures $(<140 \mathrm{mmHg})$ appeared to be harmless in that study.

A recent small-scale, cross-sectional, but deeply probing study conducted in Beer Sheva, Israel [21 $\left.{ }^{\circ}\right]$ yielded interesting findings in this regard. The team studied the outcomes of eight cognitive tests, including Mini-Mental State Examination and specific tests measuring memory, concentration and visual retention, in 495 individuals (age range 70-85 years, mean age 76.5 years) in relation to repeatedly assessed blood pressure status. They 
categorized four blood pressure groups: normotensive (mean blood pressure 122.6/72.6 mmHg; $n=136$ ); normalized hypertensive (mean blood pressure 126.8/ $74.5 \mathrm{mmHg} ; \quad n=74$ ); untreated hypertensive (mean blood pressure 152.5/81 $\mathrm{mmHg} ; n=103)$; and treated, uncontrolled hypertensive (mean blood pressure 158.7/ $85.4 \mathrm{mmHg} ; \quad n=172)$. The results in the different cognitive domains showed some variations. Nevertheless, the overall cognition scores suggest that in individuals aged 70 years or over a blood pressure in the order of 160/85 $\mathrm{mmHg}$, particularly in those receiving antihypertensive treatment, confers optimal preservation of cognitive functioning. Taking into account all findings reported above one is bound to recognize the existence of a J-curve appearing late in life, indicating a measure of reversal in the originally positive relationship between overt hypertension and dementia risk. As a consequence, antihypertensive drug treatment should be attentated when blood pressure levels tend to decline below levels in the order of 160/85 mmHg.

\section{Effects of antihypertensive drug regimes on cognitive decline}

Favourable effects of various antihypertensive drugs have been reported in clinical, partly therapeutic, followup studies on a post-hoc basis [15,22]. The effects of treatment appeared to be favourable but the findings failed to achieve statistical signficance, as summarized in a previous review [2].

A recent study conducted in Indiana, USA [23] was based on administrative data from 1900 older (age $>65$ years) nondemented hypertensive African-Americans, with a follow-up period of 5 years. In this population sample, 288 individuals (15.2\%) developed cognitive impairment. The use of antihypertensive medications overall reduced the chances of developing dementia by $38 \%$ (odds ratio $0.62,95 \%$ confidence intervals $0.48-$ 0.84). However, a corresponding analysis using reported blood pressure measurements was inconclusive. Moreover, none of the seven antihypertensive drug categories evaluated separately achieved significant reductions in odds ratios.

These partially promising estimates of the ability of antihypertensive drug treatment to preserve cognition in older hypertensive individuals could not be confirmed by findings in specifically studied subpopulations from properly randomized, placebo-controlled, prospective trials. None of the Systolic Hypertension in the Elderly (SHEP) [24], the Medical Research Council [25] or the Systolic Hypertension in Europe (Syst-Eur) [26] trialists was able to detect significant cognitive benefits from the use of active drugs (diuretics, $\beta$-blockers or a calcium channel blocker) over their respective placebos, despite significant net reductions in blood pressure (mainly systolic). However, such a negative message need not necessarily preclude a possible effect on the occurrence of dementia as a predefined end-point.

\section{Prevention of dementia by antihypertensive regimes}

Dementia was employed as a secondary end-point in four major, long-term, placebo-controlled, randomized trials conducted in older hypertensive patients.

In the SHEP trial substudy on 2034 individuals with isolated systolic hypertension [24], the incidence of dementia after 5 years was $1.9 \%$ in the placebo group and $1.6 \%$ in the actively treated (primary drug chlorthalidone; not significant). According to a recent and more detailed evaluation [27], a differential dropout and loss of data from participants in the actively treated and placebo groups may well have biased the analysis of cognitive effects of active treatment, and might therefore have obscured a significant protective action of the antihypertensive medication.

After a median period of 2 years, The Syst-Eur trial [26] demonstrated that active calcium channel blocker (nitrendipine) based treatment reduced the incidence of dementia by $50 \%$ compared with placebo $(P=0.05)$. The number of incident cases was admittedly modest (21 versus 11 cases). However, after the completion of the double-blind phase of the trial, all patients, including those originally randomized to placebo, were offered further treatment with the active trial medication. This unique modification of conventional trial design was based on ethical and complementary considerations regarding the safety of longer-term use of nitrendipine. As far as was possible, monitoring during this secondary open phase was continuous [28 $8^{\bullet}$. As a result both the median period of observation and the incidence of dementia were doubled from 2 to 3.9 years and from 32 to 64 incident cases, respectively. The former placebo group exhibited a somewhat higher average blood pressure (by $7.0 / 3.2 \mathrm{mmHg}$ ) than did the group who had received active treatment from the beginning of the study. The latter group maintained a similar and even more convincing reduction in incident dementia than in the previous analysis (now $-55 \% ; P<0.001$ ). Figure 1 shows the overall course of events.

In the Perindopril Protection Against Recurrent Stroke Study (PROGRESS) trial [29], the angiotensin-converting enzyme inhibitor perindopril failed to prevent dementia. However, its combination with the diuretic indapamide resulted in $23 \%$ reduction in the incidence of dementia $(P=0.05)$. The Study on Cognition and Prognosis in the Elderly (SCOPE) [30] tested the protective effect of the angiotensin II receptor subtype 1 blocker candesartan against placebo. It should be 
Figure 1. Incidence of dementias in the Syst-Eur, and the subsequent Syst-Eur 2 trial, according to 'intention to treat' analysis

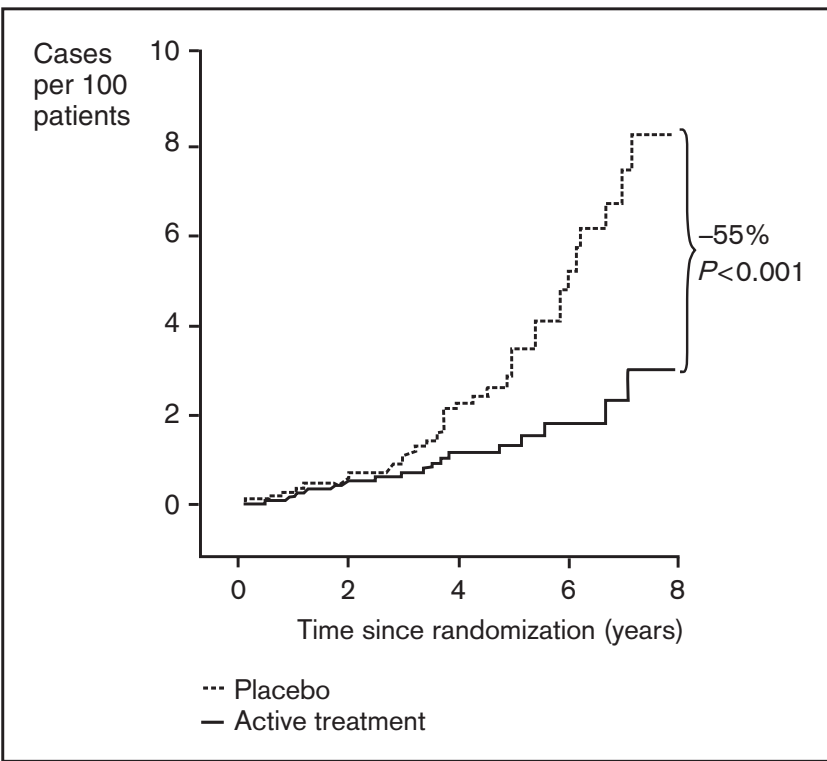

After the completion of the double-blind phase (halfway through the period of study), the original placebo group were switched to the same active (basically trial bound) medication as the group randomly assigned to active treatment from the start of the trial. The results are clearly in favour of earlier (and more effective) active nitrendipine based treatment. Adapted with permission from Forette et al. [28].

noted that $80 \%$ of patients in the 'placebo' group were actually being treated with a diuretic. No protective effect of candesartan on dementia could be demonstrated.

Taken together, these four heterogeneous trials yielded an insignificant outcome with respect to the pooled odds ratio $(0.89,95 \%$ confidence interval $0.75-1.04)$ [6]. This inconclusive result was much dependent on the use of drugs affecting the renin-angiotensin system. Exclusion of the latter (perindopril without indapamide, candesar$\tan )$ leads to a more promising pooled odds ratio $(0.75$, confidence intervals $0.60-0.94)$ [6].

\section{Discussion}

The central issue in the present review was to examine the effects of currently available antihypertensive drugs in terms of their potential additive benefit (i.e. on top of their already established cardiovascular preventive effects) with regard to the generally predictable course of cognitive decline and incidence of vascular and degenerative dementias in ageing hypertensive persons.

Before commenting on the overall findings of the trials we feel compelled to raise a caveat regarding clinical practice. According to recently extended findings, the stereotypical straightforward relationship between blood pressure elevation and cognitive malfunction appears to take on a 'J-shaped' configuration very late in life. The potential occurrence of such a 'J-curve' mandates careful and regular monitoring of blood pressure during that ultimate phase, particularly in those patients who are responsive to treatment, in order to prevent relative hypotension as a potential factor that worsens cognitive failure.

Although arbitrary antihypertensive treatment efforts in clinical practice suggested favourable effects on cognitive decline in responsive hypertensive patients, such statistically unsupported findings could not be confirmed in randomized, placebo-controlled trials. Nevertheless, to a varying extent overt dementia was found to be preventable by combining the results of three (arms of) trials (SHEP, PROGRESS and Syst-Eur), resulting in a pooled odds ratio of 0.75 (95\% confidence intervals $0.60-094 ; P=0.01)$. It appeared that the use of the dihydropyridine calcium channel blocker nitrendipine as the primary drug in Syst-Eur was the most important component in this overall result. In the latter trial, including its open extension, the cumulative incidence of dementia during a median course of 3.9 years was reduced by $55 \%$ (Fig. 1). In our view, this cannot be dismissed as a fortuitous finding, in view of the probably fundamental role of cerebrocellular calcium surfeit as a trigger of the neurotoxic cascade, which ultimately leads to both degenerative and vascular dementias.

The protective effects against the cardiovascular sequelae of hypertension in older hypertensives are, in general, equal between the different drug classes, and overall this leads to prolonged survival. This necessitates a shift of attention toward preserving mental health in the survivors.

Because of the loss of autonomy that occurs with advanced age, the increasing size of the elderly population worldwide will pose an enormous burden of care, both regarding the direct (personal) environment and society as a whole. The prevalence of (systolic) hypertension in the ageing population is approximately $50 \%$, and hence presumably this will constitute a major proportion of those destined to become demented. This indicates a new direction for research and development, aimed at identifying and employing those antihypertensive drug categories that carry the greastest potential for delaying both degenerative and vascular dementias. According to the present limited evidence available from randomized therapeutic hypertension trials, the category of dihydropyridine calcium channel blockers, exemplified by nitrendipine, currently appears to be the most promising in this regard. Admittedly this may still be a fortuitous clinical finding. However, a range of basic 
research findings indicate that intracerebral neuronal surfeit of calcium with ageing is likely to constitute a major factor that initiates a cascade of neurotoxic effects that ultimately lead to both degenerative and vascular subtypes of dementia [31,32].

The major question is, where do we go from here? According to Frishman [3], the prevention and management of dementia are formidable public health challenges that will continue well into the 21 st cenury, because the size of the older population will double in the next 30 years. This is among the consequences of reducing cardiovascular risk by virtue of antihypertensive treatment as one of the most stringently propagated factors. Because the current antihypertensive drug categories in this respect appear to be similarly effective, they all contribute to prolongation of survival in a considerable proportion of elderly hypertensive persons. Without concomitant efficacy for the prevention of dementia, we are paradoxically left with a greater burden of dementia in the population at large.

That gloomy perspective calls for a new and compelling mission, in that the medical community should now focus on the selection of those antihypertensive drugs that specifically offer the best protection against dementia. On the basis of the evaluation of randomized, placebo-controlled trials conducted thus far, together with considerations on a cerebrocellular level [31,32], the most promising candidate drug type for a new, comparative trial would be a dihydropyridine calcium channel blocker with cerebral affinity as compared to standard antihypertensive treatment with a diuretic regimen.

Much to our regret, the pharmaceutical industry has thus far remained unresponsive to such an iniative, for undisclosed but imaginable marketing reasons. Clearly, the huge ethical responsibility for this final touch, which will hopefully lead to firm approach to prevention worldwide, should now be appropriated by public health authorities, including the World Health Organization.

\section{References and recommended reading}

Papers of particular interest, published within the annual period of review, have been highlighted as:

- of special interest

- of outstanding interest

1 Seux ML, Forette F. Effects of hypertension and its treatment on mental function. Curr Hypertens Rep 1999; 1:232-237.

2 Birkenhäger $\mathrm{WH}$, Forette $\mathrm{F}$, Seux $\mathrm{ML}$, et al. Blood pressure, cognitive functions, and prevention of dementia in older patients with hypertension. Arch Intern Med 2001; 161:152-156.

3 Frishman $\mathrm{WH}$. Are antihypertensive agents protective against dementia? A review of clinical and preclinical data. Heart Disease 2002; 4:380-386.
4 Amenta F, Mignini F, Rabbia F, et al. Protective effect of antihypertensive treatment on cognitive function in essential hypertension. J Neurol Sci 2002; 203-204:147-151.

5 Rigaud AS, Olde-Rikkert MGM, Hanon O, et al. Antihypertensive drugs and cognitive function. Curr Hypertens Reports 2002; 4:211-215.

6 Wang J-G, Staessen JA, Birkenhäger WH. Antihypertensive treatment and prevention of stroke and dementia. Semin Cerebrovasc Dis Stroke 2003; 3:155-164.

7 Hofman A, Ott A, Breteler MMB, et al. Atherosclerosis, apolopoprotein E, and prevalence of dementia and Alzheimer's disease in the Rotterdam Study. Lancet 1997; 349:151-154.

8 Neuropathology Group Medical Research Council Cognitive Function and Aging Study. Pathological correlation of late-onset dementia in a multicentre, community-based population in England and Wales. Lancet 2001; 357:169175.

9 Elias ME, Wolf PA, D'Agostino RB, et al. Untreated blood pressure level is inversely related to cognitive functioning: the Framingham Study. Am J Epidemiol 1993; 138:353-364.

10 Launer J, Masaki K, Petrovich $\mathrm{H}$, et al. The association between midlife blood pressure levels and late-life cognitive function: the Honolulu-Asia Aging Study. JAMA 1995; 274:1846-1851.

11 Skoog I, Lernfelt B, Landahl S, et al. Fifteen-year longitudinal study of blood pressure and dementia. Lancet 1996; 347:1141-1145.

12 Kilander L, Nyman $H$, Boberg M, Lithell $H$. Hypertension is related to cognitive impairment: a 20-year follow-up study of 999 men. Hypertension 1998; 31:780-786.

13 Piguet O, Grayson DA, Creasey $\mathrm{H}$, et al. Vascular risk factors, cognition and dementia incidence over 6 years in the Sydney Older Persons Study. Neuroepidemiology 2003; 22:165-171.

14 Glynn RJ, Neckett LA, Hebert LE, et al. Current and remote blood pressure and cognitive decline. JAMA 1999; 281:438-445.

15 Tzourio C, Dufouil C, Ducimetiere P, Alperovitsch A. Cognitive decline in individuals with high blood pressure. Neurology 1999; 53:1948-1952.

16 Qiu Ch, Von Strauss E, Fastbom J, et al. Low blood pressure and risk of dementia in the Kungsholmen Project. Arch Neurol 2003; 60:223-228.

17 Skoog I. Blood pressure and dementia. In: Hansson L, Birkenhager WH, eds. Assessment of hypertensive organ damage. Handbook of hypertension, vol. 18. Amsterdam: Elsevier Science; 1997. pp. 303-331.

18 De la Torre JC. Alzheimer disease as a vascular disorder: nosological evidence. Stroke 2002; 33:1152-1162.

19 Belleli G, Pezzini A, Bianchetti A, Trabucchi M. Increased blood pressure variability may be associated with cognitive decline in hypertensive elderly subjects with no dementia [letter]. Arch Intern Med 2002; 162:483-484.

20 Waldstein SR. Hypertension and neuropsychological function: a lifespan perspective. Aging Res 1995; 21:321-352.

21 Paran $\mathrm{E}$, Anson $\mathrm{O}$, Reuveni $\mathrm{H}$. Blood pressure and cognitive functioning - among independent elderly. Am J Hypertens 2003; 16:818-826.

A small-scale, but qualitatively excellent cross-sectional analysis of multiple cognitive functions in community-dwelling elderly normotensives, untreated hypertensives, normalised hypertensives and uncontrolled hypertensives. The results strongly support the theory of a J-curve in the relationship between blood pressure and cognition in aging subjects.

22 Guo Z, Fratiglioni L, Winblad B, Viitanen M. Blood pressure and performance on the Mini-Mental State Examination in the very old: cross-sectional and longitudinal data from the Kungsholmen Project. Am J Epidemiol 1997; 145:1106-1113.

23 Murray MD, Lane KA, Gao S, et al. Preservation of cognitive function with antihypertensive medications. Arch Intern Med 2002; 162:2090-2096.

24 Applegate WB, Pressels S, Wittes J, et al. Impact of the treatment of isolated systolic hypertension on behavioral variables: results from the Systolic Hypertension in the Elderly Program. Arch Intern Med 1994; 154:21542160.

25 Prince MJ, Bird AS, Blizard RA, Mann AH. Is the cognitive function of older patients affected by antihypertensive treatment? Results from 54 months of the Medical Research Council's treatment trial of hypertension in older adults. BMJ 1996; 312:801-805 
26 Forette F, Seux ML, Staessen JA, et al. Prevention of dementia in randomised double-blind placebo-controlled Systolic Hypertension in Europe (Syst-Eur) trial. Lancet 1998; 352:1347-1351.

27 Di Bari M, Pahor M, Franse LV, et al. Dementia and disability outcomes in large hypertension trials: lessons learned from the Systolic Hypertension in the Elderly Program (SHEP). Am J Epidemiol 2001; 153:72-78.

28 Forette F, Seux ML, Staessen JA, et al. The prevention of dementia with - antihypertensive treatment: new evidence from the Systolic Hypertensin in Europe (Syst-Eur) Study. Arch Intern Med 2002; 162:2046-2052.

This extended open follow-up of the (originally double-blind randomized) Dementia Project in the elderly hypertensive Syst-Eur population demonstrated a progressive benefit of early protection against dementia, by virtue of the dihydropyridine calcium antagonist nitrendipine as the mainstay of antihypertensive treatment.
29 The PROGRESS Collaborative Group. Effects of blood pressure lowering with perindopril and indapamide therapy on dementia and cognitive decline in patients with cerebrovascular disease. Arch Intern Med 2003; 163:10691075.

30 Lithell H, Hansson L, Skoog I, et al. The Study on Cognition and Prognosis in the Elderly (SCOPE): principal results of a randomized double-blind intervention trial. J Hypertens 2003; 21:875-886.

31 Thibault O, Porter NM, Chen K-C, et al. Calcium dysregulation in neuronal aging and Alzheimer's disease: history and new direction. Cell Calcium 1998; 24:417-433.

32 Pascale A, Etcheberrigaray R. Calcium alterations in Alzheimer's disease: pathophysiology, models and opportunities. Pharmacol Res 1999; 39:81-88. 